\title{
Policy consensus to make inroads toward securing a Cavity-Free Future
}

$\mathrm{T}$ he Alliance for a Cavity-Free Future (ACFF) has published a Global Consensus for achieving a dental cavity-free future, a comprehensive collection of policy recommendations that if adopted by both dental and health policymakers, will help secure the changes needed to push towards a future free from cavities.

Dental caries, the disease which leads to tooth decay when left untreated, is the most prevalent non-communicable disease in the world. It is a chronic disease that afflicts people of all ages and can affect people throughout their life. It is estimated that more than 2.3 billion people suffer from untreated caries of permanent teeth, with a further 530 million children suffering from untreated caries of primary teeth. Whilst caries does affect people from all backgrounds, children and adults from disadvantaged backgrounds suffer disproportionately. Despite these stark facts, it is widely acknowledged that with appropriate measures, caries can be managed, and the resulting cavities are largely preventable.

In 2020, the Alliance for a Cavity-Free Future formed the Making Cavities History Taskforce, drawing on the collaborative effort of international experts from across dental and public health to develop a series of policy recommendations to change the way dental caries is managed. The results of this group's hard work were published in March 2021 in A Global Consensus for achieving a dental cavity-free future.

The Consensus lays out clear recommendations for policy makers at both the Global and National levels. If implemented, these policies would ensure effective caries prevention and management and offers the best opportunities to see improvements in oral and overall health. The recommendations cover the full scope of caries management to include both public and professional education, diet and nutrition, a shift to preventive dental medicine and comprehensive data collection to monitor the issue.

Since publication, the Consensus has been widely circulated, and acts to support the WHO 2021 Resolution on Oral Health which was adopted by the World Health Assembly in May 2021. The consensus report has since been endorsed by international organisations including the World Federation of Public Health Associations, reflecting the importance of the Consensus' message that collaboration across all of public health is vital to successfully tackling the disease. Recently, the British Dental Journal has published an editorial on the Consensus and the issue of dental caries as a whole.

Professor Nigel Pitts, Chairman of the ACFF, commented: 'Caries is a preventable disease that affects billions of people. The global response to the Consensus has already been hugely positive. Alongside the WHO 2021 Resolution on Oral Health, the policies laid out in the Consensus have the potential to radically impact the way caries is managed around the world and to change people's lives for the better'

To find out more about the Consensus visit www.acffglobal. org/makingcavities-history/.
Dentists and Which? warn of risks from buying teeth whitening products online

The British Dental Association has long advised the public to be wary of teeth whitening products bought online or over-the-counter for safety reasons, and welcomes new analysis by Which? that alarmingly reveals that some whiteners tested contained 300 times the legally permissible amount of hydrogen peroxide.

The Which? report highlights that two products the consumer body tested both teeth whitening gel-filled syringes bought from sellers on AliExpress contained dangerously high levels of hydrogen peroxide: around 30\% hydrogen peroxide or 300 times the amount that can be legally sold directly to consumers on the high street or online.

The Which? report also reveals that 21 of the 36 teeth whiteners tested from online marketplaces contained more than the legal amount of hydrogen peroxide $(0.1 \%)$ permitted for home use in teeth whitening kits. 11 of the 36 contained more than $6 \%$ hydrogen peroxide, which is the limit in terms of what dentists are allowed.

While the offending products have been removed from online sellers, we agree with Which? that these findings raise further questions over the checks and monitoring carried out by online marketplaces. BDA board member, Paul Woodhouse, who features in the Which? video, warns the public of the dangers of buying teeth whiteners that fail to declare their precise chemicals and may cause permanent damage to teeth. He said: 'Hydrogen peroxide is a seriously strong chemical and not to be messed with.

'Dentists are trained in its usage and they also know what whitening products are effective to use and safe.

'If you destroy gum tissue, you are never going to get it back and you lose your teeth. If it penetrates the surface of your tooth, which is likely, it's probably going to lead to the death of that tooth. 